Journal Club

Editor's Note: These short, critical reviews of recent papers in the Journal, written exclusively by graduate students or postdoctoral fellows, are intended to summarize the important findings of the paper and provide additional insight and commentary. For more information on the format and purpose of the Journal Club, please see http://www.jneurosci.org/misc/ifa_features.shtml.

\title{
Yet Another Role for SIRT1: Reduction of $\alpha$-Synuclein Aggregation in Stressed Neurons
}

\author{
Nynke Oosterhof, Dorine W. Dekens, Tjitske F. Lawerman, and Mirjan van Dijk \\ Department of Neuroscience, University Medical Centre Groningen, Graduate School for Behavioral and Cognitive Neurosciences, University of Groningen, \\ 9713 AV Groningen, The Netherlands \\ Review of Donmez et al.
}

Inclusions containing $\alpha$-synuclein are considered the major pathologic hallmark of a subgroup of neurodegenerative diseases, including Parkinson's disease (PD) and Lewy body dementia. In these so-called synucleinopathies, $\alpha$-synuclein (normally a tetrameric soluble protein) appears as oligomers and aggregates. Point mutations or gene multiplications of $\alpha$-synuclein are associated with familial forms of synucleinopathy. $\alpha$-Synuclein is mainly localized in presynaptic terminals in the brain and is thought to play a key role in synaptic functions. It has become widely accepted that abnormal $\alpha$-synuclein metabolism plays a key role in the neurodegenerative processes in synucleinopathies. Although the precise underlying mechanisms remain unknown, protein misfolding and dysfunction of the ubiquitinproteosome pathway, facilitated by agerelated mitochondrial dysfunction and oxidative stress, are thought to be involved in $\alpha$-synuclein pathogenesis.

Formation and accumulation of toxic aggregates in cells are controlled by molecular chaperones that either refold or promote degradation of misfolded proteins by the ubiquitin-proteosome pathway. Heat shock proteins (HSPs) are the most prominent molecular chaperones

Received Feb. 28, 2012; revised March 26, 2012; accepted March 28, 2012. We thank Dr. J. C. V. M. Copray for his advice and assistance.

Correspondence should be addressed to Mirjan van Dijk, Laan van de Vrede

99, 9728 CE Groningen, The Netherlands. E-mail: m.van.dijk.31@ student.rug.nl.

DOI:10.1523/JNEUROSC1.0959-12.2012

Copyright $\odot 2012$ the authors $\quad 0270-6474 / 12 / 326413-02 \$ 15.00 / 0$ controlling neurodegeneration-related aggregates. In particular, HSP70 has been shown to reduce the number of $\alpha$-synuclein aggregates (Klucken et al., 2004). Transcription of HSP70 under heat shock conditions is controlled by heat shock factor 1 (HSF1), which must be deacetylated before it can bind and activate the $h s p 70$ promotor (Westerheide et al., 2009). Sirtuins are NAD-dependent deacetylases that are thought to effectuate the deacetylation of HSF1 in the heat shock response.

Donmez et al. (2012) hypothesized that SIRT1, a sirtuin that suppresses $\beta$-amyloid production and tau protein aggregation (Min et al., 2010), might also be involved in the HSF1-HSP70-mediated reduction of $\alpha$-synuclein aggregation. They studied the role of SIRT1 in $\alpha$-synuclein aggregation and neuroprotection using the following three strains of transgenic mice: (1) mice with an A53T mutation in the $\alpha$-synuclein gene, a mutation also found in familial cases of PD; (2) mice overexpressing SIRT1; and (3) mice with brain-specific knockout of SIRT1. By crossing these strains, they obtained A53T mice, which either had SIRT1 overexpression or lacked SIRT1 activity in the brain. A53T mice overexpressing SIRT1 had a significantly longer lifespan than A53T mice, whereas A53T mice lacking brain SIRT1 activity had a significantly shorter lifespan, indirectly suggesting that SIRT1 may be neuroprotective.

Immunohistochemical analysis revealed the presence of $\alpha$-synuclein aggre- gates in the cortex and the brainstem of A53T mice starting from 2.5-3 months of age; no $\alpha$-synuclein aggregates were detected in wild-type mice. The number of $\alpha$-synuclein aggregates was significantly lower in adult $A 53 T$ mice overexpressing SIRT1 and significantly higher in A53T mice lacking SIRT1, compared with A53T mice. This suggests that SIRT1 reduces $\alpha$-synuclein aggregate formation in brain of $A 53 T$ mice.

To examine whether HSF1 deacetylation and subsequent HSP70 expression might be the underlying mechanism in SIRT1mediated $\alpha$-synuclein aggregate reduction, Donmez et al. (2012) analyzed whole-brain extracts of the different transgenic mice strains. Acetylation of HSF1 in A53T mice was significantly elevated compared with wild-type mice. However, A53T mice that overexpressed SIRT1 showed deacetylation of HSF1, reaching wild-type HSF1 acetylation levels. Since there is little acetylation in wild-type mice, mutated $\alpha$-synuclein seems to be the trigger for HSF1 acetylation. Moreover, whole-brain HSP70 RNA levels were significantly increased in A53T mice overexpressing SIRT1 and significantly decreased in SIRT1-null A53T mice relative to A53T controls.

Next, Donmez et al. (2012) investigated the interaction between SIRT1 and HSF1 in more detail, and subjected mouse embryonic fibroblasts (MEFs) from wildtype mice, from SIRT1 overexpressing mice, and from SIRT1 knock-out mice to heat shock. Analysis of the immunopre- 
cipitated protein complexes from cell lysates revealed that SIRT1 bound to and deacetylated HSF1 only after heat shock, suggesting that an interaction between these two proteins can only occur under such stress conditions. MEFs that overexpressed SIRT1 had significantly lower levels of HSF1 acetylation after heat shock than wild-type and SIRT1 knock-out MEFs. Consequently, and also only in response to heat shock, SIRT1-overexpressing MEFs had higher levels of HSP70 protein than wild-type MEFs.

Finally, Donmez et al. (2012) studied $\alpha$-synuclein aggregate-related toxicity and the role of SIRT1 in H4 cells, a human neurogliomal cell line that conditionally expressed A53T $\alpha$-synuclein. Induction of A53T $\alpha$-synuclein expression resulted in increased cell death after 48 h. Overexpression of SIRT1 or HSP70 decreased toxicity, whereas silencing SIRT1 or HSP70 increased toxicity. SIRT1 overexpression in HSP70-silenced H4 cells resulted in a smaller increase in toxicity. These data suggest that SIRT1 and HSF1 use the same pathway to decrease A53T-associated toxicity.

In summary, Donmez et al. (2012) showed a link between SIRT1-mediated deacetylation of HSF1 and A53T $\alpha$-synuclein. They demonstrated that SIRT1 deacetylates HSF1 in neurons stressed by the presence of mutated $\alpha$-synuclein and subsequently deacetylated HSF1 activates HSP70 transcription, and that increased levels of HSP70 subsequently are accompanied by a reduction in $\alpha$-synuclein aggregate formation. Donmez et al. (2012) concluded that SIRT1 protects neurons against $\alpha$-synuclein aggregateassociated toxicity via HSP70 and, therefore, that SIRT1-activating drugs may be effective in neuroprotective therapies.

Although the results of Donmez et al. (2012) support their conclusion, it is still possible that other heat-shock proteins are involved in the neuroprotective effects of SIRT1. They focused on expression of HSP70 because several studies have suggested involvement of HSP70 in aggregation reduction (Klucken et al., 2004). However, another study was unable to demonstrate a beneficial effect of HSP70 in mice with $\alpha$-synucleinopathy (Shimshek et al., 2010). Furthermore, Westerheide et al. (2009) showed that SIRT1 influences expression of other heat shock proteins, for instance HSP27, which has protective activity too (Beere, 2005).

Moreover, the therapeutic value of reducing $\alpha$-synuclein aggregates is also uncertain, given that the role of large aggregates as the key cell pathogenic mechanism of $\alpha$-synuclein is still debated. Several studies have shown that oligomeric $\alpha$-synuclein molecules may cause toxicity by perforating mitochondrial membranes (van Rooijen et al., 2010). Other studies even imply that $\alpha$-synuclein aggregation might be cytoprotective (Takeda et al., 2006). Although Donmez et al. (2012) report the correlation of SIRT1-mediated $\alpha$-synuclein aggregate reduction with prolonged lifespan, no clear evidence for a causal relation among $\alpha$-synuclein aggregate formation, cell loss, and shorter lifespan was shown. Other mechanisms besides the presence of large $\alpha$-synuclein aggregates may have accounted for cell loss and the reduction in lifespan, and accordingly SIRT1 may have had a different effect.

It is also important to note that SIRT1 has a wide range of targets and is involved in numerous cellular processes and functions. Examples include regulation of energy metabolism, cellular stress resistance, circadian clock rhythm, tumor suppression, and tumorigenesis (Finkel et al., 2009). Not all pathways regulated or featured by SIRT1 are completely understood. For example, only recently it was found that SIRT1 may deacetylate histone methyltransferases and histone proteins, and thereby regulate epigenetic silencing and chromatin modification (Finkel et al., 2009). Moreover, it has become clear that SIRT1 does not exert its effects only by deacetylating target proteins: SIRT1-mediated neuroprotection in cerebellar granule neurons is independent of deacetylation (Pfister et al., 2008). Thus, the effects of SIRT1 either on the reduction of $\alpha$-synuclein aggregate formation and/or lifespan extension in mice expressing mutated $\alpha$-synuclein may have been the consequence of SIRT1 actions mediated via other pathways.

Finally, the proposed activation of SIRT1 as a possible therapeutic strategy in the treatment of $\alpha$-synuclein aggregation still needs further investigation. If SIRT1 activation is to be incorporated into an effective therapy, one must first find ways to avoid potential side effects. Indeed, SIRT1 overexpression had a protective effect in the study of Donmez et al. (2012). Li et al. (2008), however, described that SIRT1-inhibition can be neuroprotective by reducing IGF-I/IRS-2/RAS/ERK1/2 signaling. Furthermore, SIRT1 overexpression in mouse forebrain was seen to disturb lipid/glucose metabolism and motor function (Wu et al., 2011). In other non-neuronal cell types, SIRT1 overex- pression may induce many more unexpected side effects.

In conclusion, the study of Donmez et al. (2012) has extended the well described cellular multifunctionality of SIRT1 with a role in the reduction of $\alpha$-synuclein aggregation in neurons that were stressed by the expression of mutated $\alpha$-synuclein. However, it is crucial to examine in detail how this SIRT1 action actually protects the neurons and leads to the elongation of the lifespan of mice carrying the mutated $\alpha$-synuclein gene, before it can be considered for neuroprotective drug development.

\section{References}

Beere HM (2005) Death versus survival: functional interaction between the apoptotic and stress-inducible heat shock protein pathways. J Clin Invest 115:2633-2639.

Donmez G, Arun A, Chung CY, McLean PJ, Lindquist S, Guarente L (2012) SIRT1 protects against $\alpha$-synuclein aggregation by activating molecular chaperones. J Neurosci 32:124-132.

Finkel T, Deng CX, Mostoslavsky R (2009) Recent progress in the biology and physiology of sirtuins. Nature 460:587-591.

Klucken J, Shin Y, Masliah E, Hyman BT, McLean PJ (2004) Hsp70 reduces alpha-synuclein aggregation and toxicity. J Biol Chem 279:25497-25502.

Li Y, Xu W, McBurney MW, Longo VD (2008) SirT1 inhibition reduces IGF-I/IRS-2/Ras/ ERK1/2 signaling and protects neurons. Cell Metab 8:38-48.

Min SW, Cho SH, Zhou Y, Schroeder S, Haroutunian V, Seeley WW, Huang EJ, Shen Y, Masliah E, Mukherjee C, Meyers D, Cole PA, Ott M, Gan L (2010) Acetylation of tau inhibits its degradation and contributes to tauopathy. Neuron 67:953-966.

Pfister JA, Ma C, Morrison BE, D'Mello SR (2008) Opposing effects of sirtuins on neuronal survival: SIRT1-mediated neuroprotection is independent of its deacetylase activity. PLoS One 3:e4090.

Shimshek DR, Mueller M, Wiessner C, Schweizer T, van der Putten PH (2010) The HSP70 molecular chaperone is not beneficial in a mouse model of alpha-synucleinopathy. PLoS One 5:e10014.

Takeda A, Hasegawa T, Matsuzaki-Kobayashi M, Sugeno N, Kikuchi A, Itoyama Y, Furukawa K (2006) Mechanisms of neuronal death in synucleinopathy. J Biomed Biotechnol 2006: 19365.

van Rooijen BD, Claessens MM, Subramaniam V (2010) Membrane interactions of oligomeric alpha-synuclein: potential role in parkinson's disease. Curr Protein Pept Sci 11:334-342.

Westerheide SD, Anckar J, Stevens SM Jr, Sistonen L, Morimoto RI (2009) Stress-inducible regulation of heat shock factor 1 by the deacetylase SIRT1. Science 323:1063-1066.

Wu D, Qiu Y, Gao X, Yuan XB, Zhai Q (2011) Overexpression of SIRT1 in mouse forebrain impairs lipid/glucose metabolism and motor function. PLoS One 6:e21759. 